\title{
Collaborative Spaces as Mediators for Information Sharing in Collaborative Networks
}

\author{
António Lucas Soares ${ }^{2}$ and Fábio Alves ${ }^{1,2}$ \\ ${ }^{1}$ INESC Porto, Campus da FEUP, Rua Dr. Roberto Frias, 378, 4200-465 Porto, Portugal \\ 2 DEI, FEUP, University of Porto, Rua Dr. Roberto Frias, sn 4200-465 Porto, Portugal \\ ${ }^{3}$ ESTGF-IPP, Apartado 2054610 - 156, Felgueiras, Portugal \\ \{Asoares, fabio.j.alves\} @inescporto.pt
}

\begin{abstract}
Information and knowledge sharing within collaborative networks stills being a challenging problem. Particularly in self governed or mediated networks the information/collaboration deadlock is likely to occur if there are not instrumental methods, socially accepted, that foster usable and useful patterns of collaborative information management. This paper describes how the vision for a solution to this problem was developed using the design science frameworks and the concept of technological rules. The result is materialised in the concept collaborative spaces as pivoting collaborative structures in the network enabling locally shared information to feed the network global level.
\end{abstract}

Keywords: collaborative spaces, design science, technological rules.

\section{Introduction}

Collaboration, social networking, information and knowledge management are common places nowadays when the subject is collaborative networks of organisations for example for business applications. In the last decade thousands of research pages have been written about this, be it addressing the study of real world phenomena or proposing unforeseen strategies and tools. Nevertheless, no one dares to claim that there are enough models, architectures, theories, empirical knowledge, IT platforms, that don't justify more research on those subjects. The technological, economic, social, cultural, political context is always evolving, bringing new problems and opportunities to collaboration and networking. In spite of this, companies still need to cooperate, not just to compete, still need to work together with others, not just to develop their own products and services, still need to share knowledge, not just to increase their competitive edge. In an internet of services the need for belonging to collaborative networks is even more pressing, as the value added for the customers passes more and more for the provision of integrated, combined services. The research reported in this paper, addresses both the design of a collaborative network as a socio-technical artefact, and its outcome in a form of an information/ knowledge collaborative portal based in a social network platform. Section 2 of the paper describes the design science framework for the visioning of the platform features, 
while the section 3 details the connections between technological rules and the platform's innovative features. This research carried out during the h-know EU project ${ }^{1}$.

\section{The Socio-technical Design of a Collaborative Network}

\subsection{The Design Science Research Framework}

The design of a collaborative platform aiming to support a collaborative network of organisations is unquestionably a socio-technical process. It requires a comprehensive visioning and methodological approach addressing the business, technology, management and people dimensions. If the socio-technical artefact is being developed as part of a research and development project, or within an innovation process, its vision is normally centred around two aspects: (i) Recognised and community validated business needs (for problem solving or for grabbing a business opportunity) encompassing technology as well as social and organisational development; (ii) Informed arguments justifying a new idea or web of ideas that hopefully will end up in an original and innovative socio-technical system (with varying degrees of novelty in each of the sub-systems).

Although these aspects are intertwined, this paper addresses the later, considering, in this case, the socio-technical artefact as composed by an IT based collaborative platform, a set of procedures and methods for the network formation, operation and dissolution, as well as community (virtual breeding environment) management, and a set of relationships between the individuals, groups and organisations using the platform.

Within the Design Science paradigm, as defined by [1] for research in management, "the mission of a design science is to develop knowledge for the design and realisation of artefacts, i.e. to solve construction problems, or to be used in the improvement of the performance of existing entities, i.e. to solve improvement problems". Both the collaborative networks and the information systems (IS) engineering disciplines can be, to a certain extent, considered design science disciplines.

There are several theoretical and methodological perspectives regarding the use of design science principles in IS design [2]. In this paper the general framework proposed by [3] will be used, together with the concept of technological rules as interpreted by [1]. The goal is to show how research results from explanatory sciences research - social networks modelling and simulation in this case - can be used to inform and inspire the design.

\subsection{Getting Inspiration and Guidance}

[3] Information Systems Research Framework (ISRF) proposes that IS research (i) develops/builds theory and artifacts and (ii) justifies/evaluates the outcomes of (i) by analytical, case study, experimental, field study and simulation means. Research in IS

\footnotetext{
${ }^{1}$ http: //www.h-know.eu
} 
must be relevant for a business environment and rigourous for a scientific community. As for the relevance, ISRF aggregates in "business needs" the requirements of the business "environment" composed by people, organizations and technology. In terms of rigour, research activities are to be informed by a knowledge base of foundations (theories, frameworks, models, instruments, etc.) and methodologies (formalisms, validation criteria, data analysis, etc.). At the end of a research, its results are to be applied in the business environment and contributions are made to enrich the knowledge base.

The h-know research was guided by the above framework (eventhough sometimes not explicitly). A socio-technical artefact was developed and theory about collaboration and information/knowledge management was built. A subset of the above results will be analysed below: the architecture of the h-know collaborative platform and a theory on the relationship between information/knowledge sharing and social networking based collaboration.

For the detailed development of the informed argument leading to a web of innovative ideas (see previous section) the concept of technological rule was used. [1] defines a technological rule (after the original concept coined by the philosopher Mario Bunge) as "a chunk of general knowledge, linking an intervention or artefact with a desired outcome or performance in a certain field of application". In the case of the h-know research, the desired outcome or performance was, in general terms, the successful formation and operation of a collaborative network of SMEs and research centres, and, more specifically, to achieve effective sharing of information and knowledge in the network through effective collaboration processes.

Technological rules can be derived from explanatory sciences such as sociology, cognitive sciences, or organizational science. For the design of socio-technical solutions for business problems involving organizational work, the more useful technological rules are likely to be derived from empirical studies. For example, [4] in their study of knowledge sharing barriers in complex, multi-national RTD projects, found that one the barriers most mentioned by project managers was the lack of initiative and strategy by the workers in what concerns to organising and disseminating non-operational information, e.g., lessons learnt. The reasons that were pointed out had to do basically with lack culture of collaborative work and knowledge sharing. It is then possible to derive technological rules from this study such as: "if you want to achieve effective information sharing in a collaborative network, select the most autonomous and collaboration prone employees" or "if there is a need to control and track information effectively in a collaborative network, procedures and workflows must be put in place to prevent document exchange through email". In this paper, rules derived from empirical studies or similar are named type 1 technological rules.

However, when looking for innovative ideas to incorporate in a socio-technical artefact, besides empirical studies it might be interesting to look at experimental studies, for instance modelling and simulation studies of social structures (social networks). For example, [5] in their network models for social influences processes hypothesise that various networks may be involved in the transmission of influence in different ways, and that influence might occur not only through public knowledge but also through private dyadic interactions. This can lead to a technological rule stating 
that "the existence of private spaces for collaboration in a collaborative platform are likely to result in the global spread of collaborative practices in the network". Such a derivation of technological rules is exploratory and speculative, but it can be inspirational for discovering innovative solutions. Rules derived from non-empirical studies such the ones referred above are called here type 2 technological rules.

\section{3 h-know: A Socio-Semantic Collaborative Platform}

\subsection{Business Requirements and Scientific Objectives}

h-know research aimed to develop a collaborative network artefact (an IT based collaborative platform and a methodology for its management) responding to specific business needs from the rehabilitation, restoration and maintenance areas in the construction industry. Furthermore, as part of an EU RTD research project, there was a strong requirement for developing a solution that is placed beyond the state-of-theart, i.e., with an high degree of originality and innovativeness. Only short summary of the business needs or requirements is given next, as the focus of this paper is on the innovative requirements and solution features specification.

The increasing number of complex works in construction industry, particularly for the retrofitting, refurbishment and maintenance of old buildings or construction complexes, are urgently requesting an new solutions to enable a systematic and effective access to the construction knowledge related to this area, and to the state-ofthe-art processes and materials to be applied. In the complex world of the restoration works there is still a sub-optimality of the information flow between different actors taking a role in the processes, such as the final users of the building, the administration responsible for the restoration works and other administration involved, the architects, the bodies in charge of the diagnosis of the building, the construction companies, and the SME's in charge of specific works. This is even more complex if multi-disciplinarity required for restoration works (including archaeologists, architects, engineers, etc) is considered. For this reason, innovative solutions to support new forms of collaborative knowledge and business networks of groups of SMEs and RTD centres, experts for restoration/maintenance, are needed. There is thus a need for a solution which will provide (i) innovative competitive knowledge and training providing services, and (ii) an advanced support for realisation of new forms for SME-RTD networking through their specific knowledge/competence integration, within a new collaboration structure.

\subsection{Building a Vision for h-know}

A set of type 2 technological rules were derived during the problem definition and requirements elicitation phase. Here follow three examples:

TR_T2_03: "If the achievement of high levels of information creation and sharing in the collaborative network is fundamental, private interaction spaces should be provided for specific and situation dependent activities"; 
TR_T2_04: "If we want to increase the participation of individuals and organizations in the collaborative network, then promote the participation of influential actors (high centrality) in more than one collaboration space;

TR_T2_05: "If effective results are required from collaboration mediated by the platform, then information management support tools must be tightly coupled with social networking features";

TR_T2_06: "If we want to achieve and maintain a critical mass of members in the collaborative network, then the platform must represent an "obligatory passage point" for individuals and organisations, either by means of providing authoritative information or state-of-the art tools".

Next, some examples of explanatory results conveying the knowledge that enabled the derivation of the above rules are given. This derivation is not a systematic process, but requires interpretation and ingenuity as well as the skills to know and access relevant scientific and technical information sources.

To the socio-technical approach purposes, [6] agree that "social interaction ties are regarded as one of the antecedents in motivating knowledge sharing behaviors. The social interaction ties among individuals lead to creating trust, and wider communication, producing positive effects on sharing knowledge" and add, "The stronger social interaction ties become, the more frequent knowledge exchange behaviors as well as communication are observed" (quoted in Chai and Kim, 2011a).

[5] Social influence occurs when an individual adapts his or her behavior, attitudes or beliefs to the behavior, attitudes or beliefs of others in the social system. Influence does not necessarily require face-to-face interaction, but is based on information about other people. Social influence may arise when individuals affect others' behaviors, or when individuals imitate the behaviors of others, irrespective of the intention of the behavior's originator.

From the business requirements and the technological rules, the visioning process in the h-know research developed the concept of information and knowledge supported collaboration adopting a social network paradigm. In simple terms, h-know provides contained and private working spaces for the development of joint activities (seen as inter-organisational collaboration), strongly levered on information/knowledge management, built upon a social network platform for the interconnection of individuals and organisations.

\subsection{Collaborative Spaces: Fostering Collaboration and Information Sharing}

The concept of "collaborative space" emerged as the central structure in the platform, both in terms of collaboration tools and context and in terms of information/knowledge management. It provides content, document and event management, information 
organisation and classification based on domain ontologies, as well as the usual tools for communication and debate (such as foruns and blogs). It is a rich structure as it incorporates privacy schemes for all the informational objects and a life-cycle based on the collaboration status (see figure 1 for the overall concept and figure 2 for the life-cycle).

Despite the central role of the collaborative spaces, h-know would not respond to the initial requirements if information (and knowledge in some extent) could not be shared at the level of the business community (virtual breeding environment). This is done through a an hybrid process of publishing/collection of the public content available in each of the collaborative spaces. In fact, this is one of the distinctive features of the h-know platform: a process from local to global sharing that is likely to improve the quantity and utility of the shared information.

The h-know platform has a semantic structure for its content description implemented through the integration of social and domain ontologies. The social component uses FOAF (Friend of a Friend) and SIOC (Semantically-Interlinked Online Communities) ontologies that enable a description of the users, spaces and their relationships. The domain ontology implements a global/local approach to ontology management, and it is used to classify the content and actors according to the work and technical domains. This approach to the semantic structure of the platform enables to obtain answers to searches such as "who wrote something about subject $\mathrm{s}$ in the form of content c". A complete account of the development of the semantic structure of h-know is given by [7].

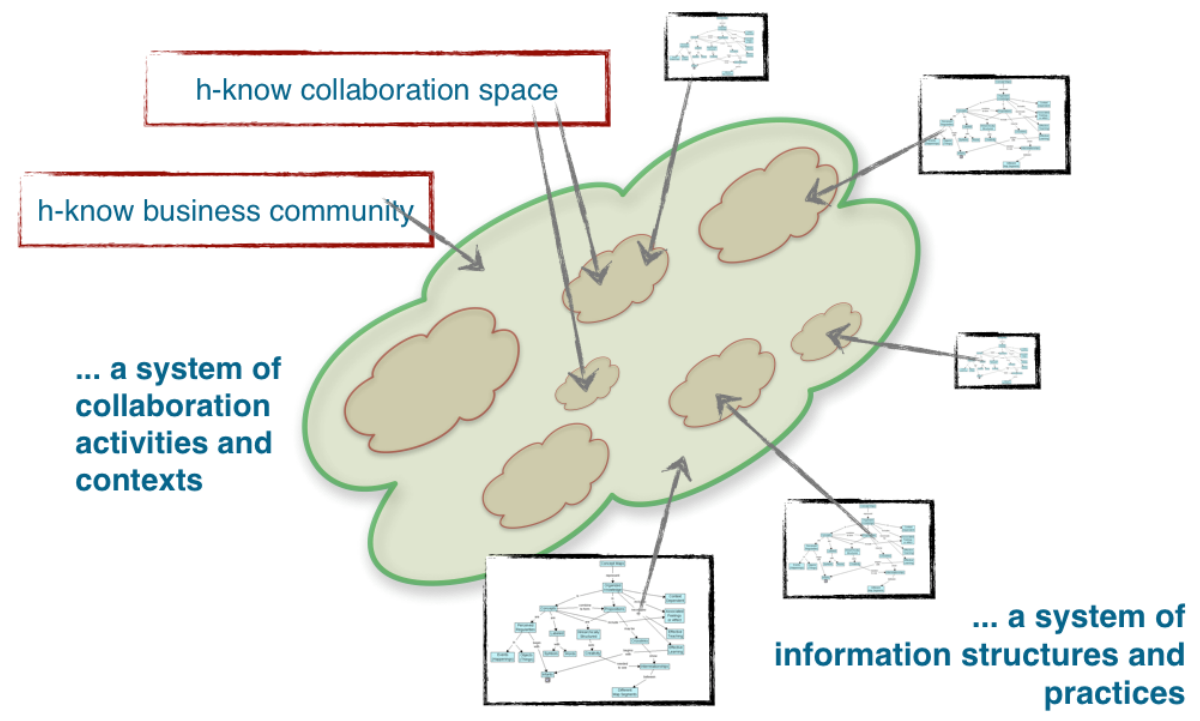

Fig. 1. The overall concept of a collaborative network structured upon collaborative spaces 


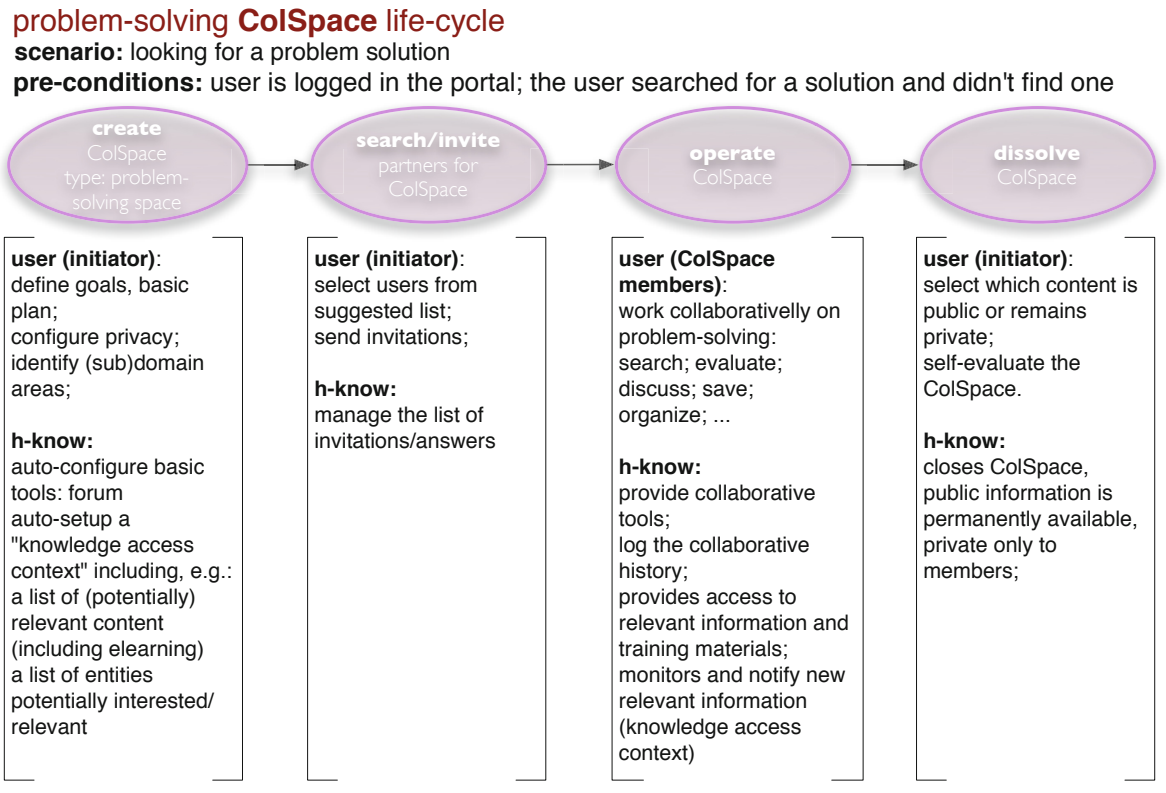

Fig. 2. An example of the life cycle of a "Problem solving type of collaborative space"

\section{Conclusions and Further Work}

h-know three years research resulted in a fully working platform ready to be used in establishing collaborative networks in a variety of domains, besides the rehabilitation, restoration and maintenance of buildings defined in the original project. In a great extent, the authors believe that this was due to the technological rules approach and the ISR framework adopted for the design of the socio-technical artefact. In this paper we did not present an evaluation/validation of the visioning process neither of the resulting h-know collaborative platform, due to space limitations. For this, the design evaluation methods proposed by [3] are the most appropriate. An informed argument and a scenario are the more adequate options in this situation, and a first approach can be found in a former paper [7]. The h-know platform is now being deployed in some real applications. There are three practical implementations planned: the implementation of a collaborative platform to support a national association of small companies in the construction sector in France, the support to a local community of small companies in the rehabilitation area in Italy (implemented through a local association) and the support to a big R\&D project in Portugal.

\section{References}

1. Van Aken, J.: Management research based on the paradigm of the design sciences: The quest for field-tested and Grounded Technological Rules. Journal of Management Studies (2004) 
2. Winter, R.: Design science research in Europe. Eur. J. Inform. Syst. (2008)

3. Hevner, A., March, S., Park, J., Ram, S.: Design Science in Information Systems Research. Mis. Quart. (2004)

4. Santos, V.R., Soares, A.L., Carvalho, J.Á.: Knowledge Sharing Barriers in Complex Research and Development Projects. Knowledge and Process Management (2012)

5. Robins, G., Pattison, P., Elliott, P.: Network models for social influence processes. Psychometrika 66, 161-189 (2001)

6. Cheng, J.-H.: Inter-organizational relationships and information sharing in supply chains. International Journal of Information Management 31, 374-384 (2011)

7. Carneiro, L.C., Sousa, C., Soares, A.L.: Integration of Domain and Social Ontologies in a CMS Based Collaborative Platform. In: Meersman, R., Dillon, T., Herrero, P. (eds.) OTM 2010 Workshops. LNCS, vol. 6428, pp. 414-423. Springer, Heidelberg (2010) 